\title{
A longitudinal cohort based association study between uric acid level and metabolic syndrome in Chinese Han urban male population
}

Qian Zhang ${ }^{1+}$, Chengqi Zhang ${ }^{2+}$, Xinhong Song ${ }^{3}$, Haiyan Lin ${ }^{3}$, Dongzhi Zhang ${ }^{3}$, Wenjia Meng ${ }^{1}$, Yongyuan Zhang ${ }^{1}$, Zhenxin Zhu' ${ }^{1}$, Fang Tang ${ }^{2}$, Longjian Liư ${ }^{4}$ Xiaowei Yang ${ }^{5}$ and Fuzhong Xue 1* $^{*^{3}}$

\begin{abstract}
Background: It has been recently demonstrated that serum uric acid (UA) is associated with metabolic syndrome (MetS) or its related clinical indications based on cross-sectional or prospective cohort studies. Nonetheless, due to the fact that UA level constantly fluctuates from time to time even for the person, using a single measure of UA level at baseline of those studies may not be sufficient for estimating the UA-Mets association.

Methods: To further estimate this time-dependent association, we fitted a generalized estimating equation (GEE) regression model with data from a large-scale 6-year longitudinal study, which included 2222 participants aged $>=25$ years with an average of 3.5 repeated measures of UA per person in the Health Management Center of Shandong Provincial Hospital, Shandong, China.

Results: After adjusting for other potential confounding factors (i.e., total cholesterol, low-density lipoprotein), it was verified that time-dependent UA level was an independent risk factor for MetS (OR=1.6920, $p<0.0001)$. It was found that UA level was positively associated with obesity, hypertension, and dyslipidemia, but was inversely associated with hyperglycemia.

Conclusions: Serum UA level may serve as an important risk factor of MetS. Additionally, our study suggested that UA level be an independent risk factor to obesity, hypertension and dyslipidemia, but a protective factor to hyperglycemia. These findings are concordant with results from other studies on Asian populations, and jointly provide a basis to further develop a risk assessment model for predicting MetS using UA levels and other factors in China.
\end{abstract}

\section{Background}

Metabolic syndrome (MetS) refers to a combination of medical disorders ,including obesity, dyslipidemia, hypertension and insulin resistance[1]. When occurring together, they increase the risk of developing cardiovascular disease (CVD) and diabetes [2]. Recent studies indicate a wider range of biomarkers of MetS, e.g., alanine aminotransferase, white blood cell count, gamma-glutamyltranspeptidase, and serum uric acid (UA) [3, 4]. Based on cross-sectional studies, both positive and negative association between UA levels and MetS has been reported [5-7]. To overcome the inherent limitation

\footnotetext{
* Correspondence: xuefzh@sdu.edu.cn

${ }^{\dagger}$ Equal contributors

'Department of Epidemiology and Health Statistics, School of Public Health, Shandong University, PO Box 100, Jinan 250012, China

Full list of author information is available at the end of the article
}

of cross-sectional design, a prospective cohort study was conducted recently, which suggested that higher serum UA level would strongly increase the risk of MetS incidence [8]. Nonetheless, such a cohort study with only baseline UA used as predictor is still limited due to the fact that UA level would vary from time to time during the lifespan of most individuals [8]. Therefore, to fully understand the relationship between UA and MetS, and further assess the specific association between UA and each medical disorder in defining MetS, longitudinal studies are expected especially those with large sample sizes and repeated measures of UA. A longitudinal study is usually a cohort study that involves repeated observations of the same set of variables over a period of time. Unlike cross-sectional studies or cohort studies with only baseline measures, a longitudinal study not only tracks each participant's outcome (e.g., MetS) but also repeatedly

\section{Biomed Central}


measures risk factors that would change with time (e.g., UA). Therefore, longitudinal studies allow one to make observations more accurately and model time-dependent relationships. They are more becoming popular in epidemiology and biomedical research. In this paper, we study the UA-MetS associations using data from a longitudinal study of male residents who visited the Health Management Center of Shandong Provincial Hospital (HMCSPH) for routine physical examinations.

\section{Methods}

\section{Study population}

In our longitudinal study, participants include 2222 male residents of Shandong Province who visited HMCSPH (an affiliate of organization of Shandong University) at least three times for annual physical examinations between 2005 and 2010. During the 6 year follow-up, each participant on average had $3.63 \pm 0.016$ measures including serum UA levels. For this study, we only included participants who had not been diagnosed as having MetS at baseline. They were all Chinese Han with baseline age between 25 and 91 years, all belong to middle and upper socioeconomic classes. This study was approved by the Ethics Committee of School of Public Health, Shandong University, and all participants gave informed written consent.

\section{Measurements}

Blood samples were collected from participants after an overnight fast of at least 12 hours. Height and weight were measured on participants wearing light clothing without shoes, and body mass index (BMI) was calculated as weight $(\mathrm{kg})$ divided by squared height $(\mathrm{m})$. Blood pressure was measured on right upper arm with participants in sitting position after 5 minutes rest. Peripheral blood samples was obtained for measuring the following parameters: glucose, total cholesterol (CHOL), low-density lipoprotein (LDL), high-density lipoprotein (HDL), triglycerides, uric acid (UA), gamma-glutamyltranspeptidase (GGT), serum albumin (ALB), serum globulins (GLO), blood urea nitrogen (BUN), serum creatinine (CREA), hemoglobin $(\mathrm{Hb})$, hematokrit (HCT), mean corpuscular volume(MCV), mean corpuscular hemoglobin $(\mathrm{MCH})$, Red blood cell distribution width (RDW), white blood count (WBC), platelet distribution width (PDW), mean platelet volume (MPV), and thrombocytocrit (PCT). Data on smoking habits, alcohol intake, diet habits, sleeping quality, exercise frequency, and other variables were obtained using standardized questionnaire.

\section{Definition of metS}

Considering the physiological characteristics of our target population, the diagnostic criteria recommended by Diabetes Branch of the Chinese Medical Association (CDS) [9] was used in defining MetS in this study. An participant was claimed as having MetS if he or she had three or more of the following four medical conditions: (1) overweight or obesity, i.e., BMI $\geq 25.0 \mathrm{Kg} / \mathrm{M}$ [2]; (2) hypertension, i.e., systolic blood pressure $(\mathrm{SBC}) \geq 140 \mathrm{mmHg}$, or diastolic blood pressure $(\mathrm{DBP}) \geq 90 \mathrm{mmHg}$, or previously diagnosed as hypertension; (3) dyslipidemia, i.e., fasting triglycerides (TG) $\geq 1.7 \mathrm{mmol} / \mathrm{L}(110 \mathrm{mg} / \mathrm{dl})$, or fasting high-density (HDL) $<0.9 \mathrm{mmol} / \mathrm{L}(35 \mathrm{mg} / \mathrm{dl})$; (4) hyperglycemia was defined as fasting blood-glucose (FPG) $\geq 6.1 \mathrm{mmol} / \mathrm{L}$ (110 mg/dl), or $2 \mathrm{~h}$ Postmeal Glucose (PG) $\geq 7.8 \mathrm{mmol} / \mathrm{L}$ (140 mg/dl),or previously diagnosed as hyperglycemia.

\section{Missing data imputation}

In our longitudinal study, there were some variables (see Table 1) with missing values due to early withdrawal of the participants or missing of certain physical examinations. To handle missing values, the strategy of multiple imputation (MI) was adopted where imputations were made using the Markov Chain Monte Carlo (MCMC) algorithm implemented in the MI Procedure of SAS 9.1.3 [10]. Then, each imputed data set was analysed using GEE regression models. Finally the multiple set of estimators for parameters of interest from the GEE analyses were combined to make final inferences. Considering that the variables in our analysis were continuously distributed with arbitrary missingness patterns, a multivariate normal distribution model was assumed in the MI Procedure. As seen in Table 1, most of the imputed variables had less than $10 \%$ of missingness.

\section{UA Levels and potential confounding factors}

Before fitting a GEE model, the original continuous serum UA measure was categorized into 4 levels using the 3 quartiles $\left(\mathrm{P}_{25}, \mathrm{P}_{50}\right.$ and $\left.\mathrm{P}_{75}\right)$ as cut-off values. As seen in Table 1 , potential confounding factors were also considered during the GEE analysis.

\section{Statistical analysis}

Descriptive analyses were first conducted for the distributions of UA levels and potential covariates collected at the baseline survey. Then, each variable of interest was compared, using student's t-test, between subjects with and subjects without MetS at each follow-up interval (i.e., each year baseline). To study the association between UA levels and MetS, we first fitted simple GEE models, each with a single predictor (i.e., the UA variable or any one of the confounding factors). We finally fitted a multiple GEE regression model. A covariate was added to the final multiple regression model only if it was found significant $(\mathrm{p}<0.05)$ in the single-predictor model. Note that the GEE modelling strategy is capable of describing timedependent relationship between UA levels and the MetS status (yes or no), after adjusting potential confounders. All these variables are repeatedly measured during the 6year follow-up. Age at baseline was also added to the 
Table 1 Definition of UA levels and potential confounding factors and missingess rate

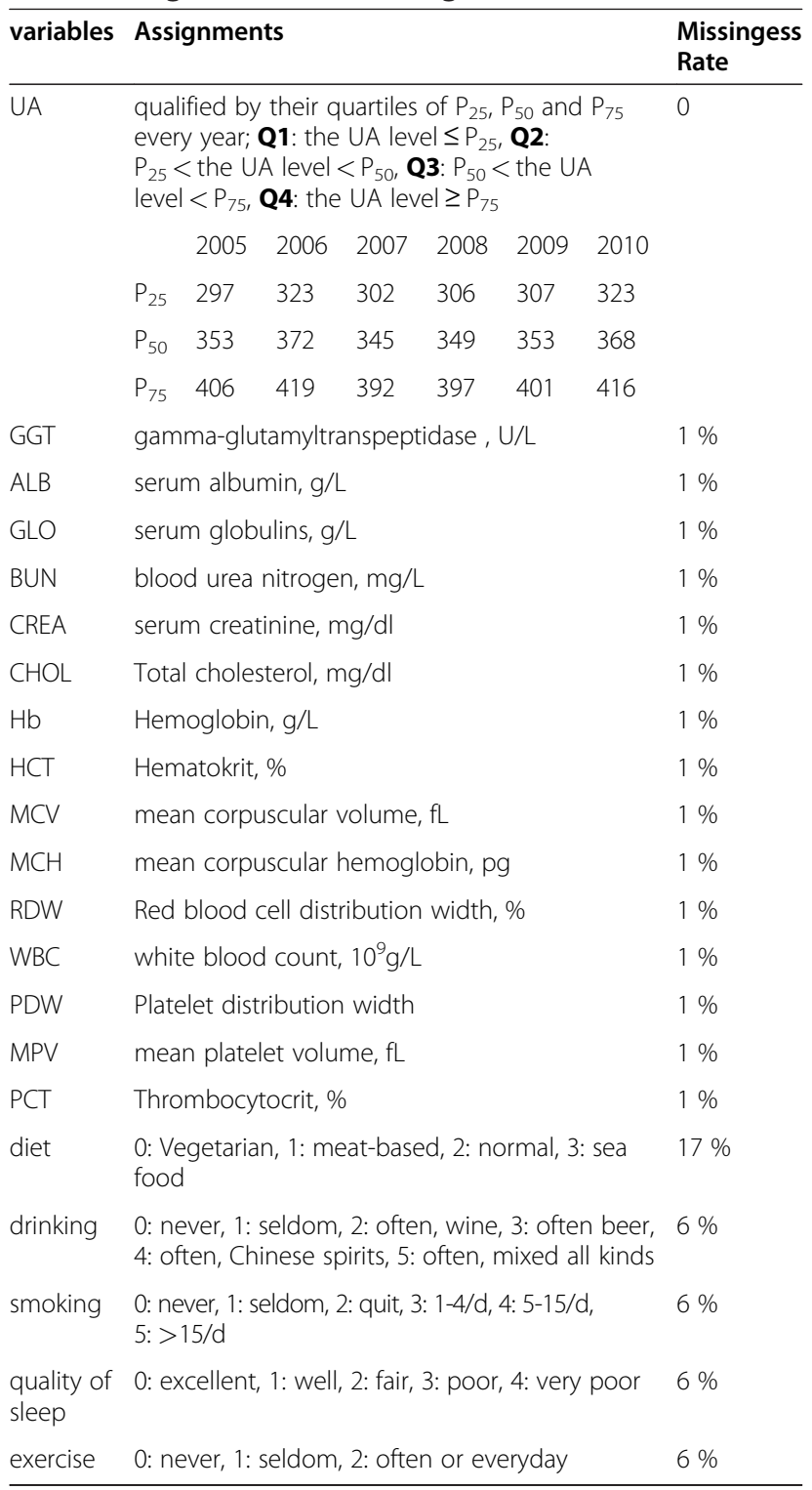

multiple GEE analysis procedure to adjust for the age effect. In each GEE model, 'Logit' was chosen as the link function of GEE, because either MetS status is a binary dependent variable. Following the same procedure, we studied the association between UA levels and each one of the four medical disorders in defining MetS (i.e., overweight/obesity, hypertension, dyslipidemia, and hyperglycemia), which were all defined as binary variables. All statistical analysis was performed by SAS 9.1.3.

\section{Results}

Additional file 1: Table S1 in Supplement Materials shows the distribution of UA levels and other potential confounding factors at the baseline survey, and their distributions between subjects with and subjects without
MetS at each follow-up interval (i.e., each year after baseline). The table shows that the UA level and other potential confounding factors in the MetS group were generally higher than their counterparts in the nonMetS group at each follow-up intervals $(\mathrm{P}<0.05)$, although some factors were not statistically significant at some follow-up intervals. The numbers of participants at each follow-up interval during the study period (Jan 2005 - Dec 2010) are also shown in the Supplemental Materials; see Additional file 1: Table S2.

Table 2 shows the results of single-predictor GEE models for the UA levels and other potential confounding factors. It is seen that the UA level was strongly associated with MetS (OR $=1.2952 ; 95 \% \mathrm{CI}=1.1864-1.4141 ; \mathrm{p}$ $<.0001)$. Age, GGT, ALB, GLO, CHOL, Hb, MCV and $\mathrm{MCH}$ and WBC were all significant predictors or confounders at the $\alpha=0.05$ level. Other factors including BUN, CREA, HCT, RDW, PDW, MPV, PCT, iet, drinking, smoking, quality of sleep and exercise are not significant at the $\alpha=0.05$ level.

Table 3 illustrates the association between UA levels and MetS status after adjusting other potential confounding factors using the final multiple GEE model. It reveals that, compared with the lowest level of UA (Q1), the highest level of UA (Q4) was strongly associated with MetS status with a positive relationship $(\mathrm{OR}=1.6920 ; 95 \% \mathrm{CI}=$ 1.3390-2.1381; $p<0.0001)$. Although not significant, the ORs for Q2 and Q3 were all larger than 1.0, and an increasing trend was seen in OR from Q2 to Q4.

The results on association of UA levels with each single MetS component, are shown in Table 4, 5, 6 and 7 of the Supplemental Materials, respectively for overweight/obesity, hypertension dyslipidemia and hyperglycemia. Table 4 shows that UA was a strong independent risk factor to overweight/obesity; and an obvious increasing trend in $\mathrm{OR}$ is seen from Q2 to Q4 after adjusting the other three components of MetS and potential confounding factors. Table 6 and 4 jointly depict similar patterns for the association between UA levels and hypertension or dyslipidemia, except that the OR is not significant at the Q2 level. In contrast, UA is identified as a protective factor with the range of ORs ranged from 0.58-0.74 after adjusting the other three components and the same set of potential confounding factors; see Table 7.

\section{Discussions}

The relationship among UA, MetS and cardiovascular diseases has received much attention in recent years. The findings from a nationwide representative sample of US adults indicate that the prevalence of the MetS increases substantially with increasing levels of UA [11]. UA as an independent risk predictor for MetS also was found in Korean male workers aged 30-39 years [12], in Thai adults receiving annual physical examinations [13], in Japanese 
Table 2 Single-predictor GEE Models

\begin{tabular}{|c|c|c|c|c|c|c|c|}
\hline & Estimate & $\begin{array}{l}\text { Standard } \\
\text { Error }\end{array}$ & Z & $\begin{array}{l}\operatorname{Pr}> \\
|Z|\end{array}$ & RR & $\begin{array}{l}\text { lower } 95 \% \\
\text { Confidence } \\
\text { Limits }\end{array}$ & $\begin{array}{l}\text { upper } 95 \% \\
\text { Confidence } \\
\text { Limits }\end{array}$ \\
\hline Quartiles of UA & 0.2587 & 0.0448 & 5.78 & $<.0001$ & 1.2952 & 1.1864 & 1.4141 \\
\hline age & 0.0243 & 0.0031 & 7.94 & $<.0001$ & 1.0246 & 1.0185 & 1.0308 \\
\hline GGT & 0.0095 & 0.0012 & 8.15 & $<.0001$ & 1.0095 & 1.0072 & 1.0118 \\
\hline ALB & 0.037 & 0.0097 & 3.8 & 0.0001 & 1.0377 & 1.0181 & 1.0576 \\
\hline GLO & -0.0574 & 0.0172 & -3.33 & 0.0009 & 0.9442 & 0.9128 & 0.9766 \\
\hline BUN & -0.0083 & 0.0431 & -0.19 & 0.8474 & 0.9917 & 0.9114 & 1.0792 \\
\hline CREA & -0.0004 & 0.0053 & -0.08 & 0.933 & 0.9996 & 0.9894 & 1.0099 \\
\hline CHOL & 0.3292 & 0.0491 & 6.7 & $<.0001$ & 1.3899 & 1.2623 & 1.5304 \\
\hline $\mathbf{H b}$ & 0.0242 & 0.0052 & 4.67 & $<.0001$ & 1.0245 & 1.0142 & 1.0350 \\
\hline $\mathrm{HCT}$ & 0.0119 & 0.0173 & 0.69 & 0.4913 & 1.0120 & 0.9782 & 1.0470 \\
\hline MCV & -0.0296 & 0.0112 & -2.64 & 0.0083 & 0.9708 & 0.9496 & 0.9924 \\
\hline MCH & 0.0905 & 0.0286 & 3.17 & 0.0015 & 1.0947 & 1.0351 & 1.1579 \\
\hline RDW & -0.0613 & 0.0674 & -0.91 & 0.3632 & 0.9405 & 0.8242 & 1.0734 \\
\hline WBC & 0.1391 & 0.0276 & 5.05 & $<.0001$ & 1.1492 & 1.0888 & 1.2130 \\
\hline PDW & 0.0045 & 0.0299 & 0.15 & 0.8795 & 1.0045 & 0.9474 & 1.0651 \\
\hline MPV & -0.0034 & 0.0623 & -0.05 & 0.9563 & 0.9966 & 0.8821 & 1.1259 \\
\hline PCT & -1.4813 & 1.0857 & -1.36 & 0.1725 & 0.2273 & 0.0271 & 1.9092 \\
\hline diet & -0.0248 & 0.0535 & -0.46 & 0.6433 & 0.9755 & 0.8784 & 1.0834 \\
\hline drinking & 0.0172 & 0.029 & 0.59 & 0.5539 & 1.0173 & 0.9611 & 1.0768 \\
\hline smoking & 0.0044 & 0.0241 & 0.18 & 0.8536 & 1.0044 & 0.9582 & 1.0530 \\
\hline quality of sleep & -0.0242 & 0.062 & -0.39 & 0.696 & 0.9761 & 0.8644 & 1.1021 \\
\hline exercise & 0.015 & 0.103 & 0.15 & 0.8841 & 1.0151 & 0.8295 & 1.2423 \\
\hline
\end{tabular}

Table 3 The results of multiple GEE analysis for UA levels and MetS after adjusting other potential confounding factors

\begin{tabular}{llllllll}
\hline & Estimate & Error & Z & Pr $>|Z|$ & RR & $\begin{array}{l}\text { lower } 95 \% \\
\text { Confidence Limits }\end{array}$ & $\begin{array}{l}\text { upper } 95 \% \\
\text { Confidence Limits }\end{array}$ \\
\hline Intercept & -5.3378 & 1.4182 & -3.76 & 0.0002 & & & \\
Q4 & $\mathbf{0 . 5 2 5 9}$ & $\mathbf{0 . 1 1 9 4}$ & $\mathbf{4 . 4 1}$ & $<.0001$ & $\mathbf{1 . 6 9 2 0}$ & $\mathbf{1 . 3 3 9 0}$ & 1.1381 \\
Q3 & 0.1856 & 0.1244 & 1.49 & 0.1357 & 1.2039 & 0.9435 & 1.3363 \\
Q2 & 0.0709 & 0.1274 & 0.56 & 0.5778 & 1.0735 & 0.8363 & ref \\
Q1 & 0 & 0 & ref & ref & ref & ref & $\mathbf{1 . 4 3 0 6}$ \\
time & $\mathbf{0 . 3 0 7 2}$ & $\mathbf{0 . 0 2 6}$ & $\mathbf{1 1 . 8 4}$ & $<.0001$ & $\mathbf{1 . 3 5 9 6}$ & $\mathbf{1 . 2 9 2 3}$ & $\mathbf{1 . 0 3 8 1}$ \\
baseage & $\mathbf{0 . 0 3 0 8}$ & $\mathbf{0 . 0 0 3 4}$ & $\mathbf{9 . 1 6}$ & $<.0001$ & $\mathbf{1 . 0 3 1 3}$ & $\mathbf{1 . 0 2 4 5}$ & $\mathbf{1 . 0 1 1 5}$ \\
GGT & $\mathbf{0 . 0 0 9 1}$ & $\mathbf{0 . 0 0 1 1}$ & $\mathbf{7 . 9 6}$ & $<.0001$ & $\mathbf{1 . 0 0 9 1}$ & $\mathbf{1 . 0 0 6 9}$ & 1.0176 \\
ALB & -0.018 & 0.018 & -1 & 0.3197 & 0.9822 & 0.9481 & $\mathbf{1 . 0 4 3 9}$ \\
GLO & $\mathbf{0 . 0 2 2 8}$ & $\mathbf{0 . 0 1 0 3}$ & $\mathbf{2 . 2 1}$ & $\mathbf{0 . 0 2 6 8}$ & $\mathbf{1 . 0 2 3 1}$ & $\mathbf{1 . 0 0 2 6}$ & $\mathbf{1 . 2 4 6 3}$ \\
CHOL & $\mathbf{0 . 1 2 8 5}$ & $\mathbf{0 . 0 4 6 8}$ & $\mathbf{2 . 7 4}$ & $\mathbf{0 . 0 0 6 1}$ & $\mathbf{1 . 1 3 7 1}$ & $\mathbf{1 . 0 3 7 4}$ & $\mathbf{1 . 0 2 1 2}$ \\
Hb & $\mathbf{0 . 0 1 2}$ & $\mathbf{0 . 0 0 4 6}$ & $\mathbf{2 . 6 3}$ & $\mathbf{0 . 0 0 8 6}$ & $\mathbf{1 . 0 1 2 1}$ & $\mathbf{1 . 0 0 3 1}$ & $\mathbf{0 . 9 0 9 5}$ \\
MCV & $\mathbf{0 . 1 3 2 1}$ & $\mathbf{0 . 0 1 9}$ & $\mathbf{- 6 . 9 6}$ & $<.0001$ & $\mathbf{0 . 8 7 6 3}$ & $\mathbf{0 . 8 4 4 3}$ & $\mathbf{1 . 4 5 7 5}$ \\
MCH & $\mathbf{0 . 2 8 6 2}$ & $\mathbf{0 . 0 4 6 2}$ & $\mathbf{6 . 1 9}$ & $<.0001$ & $\mathbf{1 . 3 3 1 4}$ & $\mathbf{1 . 2 1 6 0}$ & $\mathbf{1 . 2 2 2 0}$ \\
WBC & $\mathbf{0 . 1 5 3 1}$ & $\mathbf{0 . 0 2 4 2}$ & $\mathbf{6 . 3 3}$ & $<.0001$ & $\mathbf{1 . 1 6 5 4}$ & $\mathbf{1 . 1 1 1 5}$ & \\
\hline
\end{tabular}


Table 4 The results of multiple GEE analysis for UA levels and Obesity after adjusting other potential confounding factors

\begin{tabular}{|c|c|c|c|c|c|c|c|}
\hline Quartiles & Estimate & Error & Z & $\operatorname{Pr}>|Z|$ & RR & $\begin{array}{l}\text { lower } 95 \% \\
\text { Confidence Limits }\end{array}$ & $\begin{array}{l}\text { upper } 95 \% \\
\text { Confidence Limits }\end{array}$ \\
\hline Intercept & -0.3359 & 0.8418 & -0.4 & 0.6899 & & & \\
\hline Q4 & 0.9224 & 0.0695 & 13.28 & $<.0001$ & 2.5153 & 2.1953 & 2.8823 \\
\hline Q3 & 0.7133 & 0.0653 & 10.92 & $<.0001$ & 2.0407 & 1.7955 & 2.3194 \\
\hline Q2 & 0.4271 & 0.0634 & 6.73 & $<.0001$ & 1.5328 & 1.3536 & 1.7357 \\
\hline Q1 & 0 & 0 & ref & ref & ref & ref & ref \\
\hline hypertension & 0.1446 & 0.0581 & 2.49 & 0.0128 & 1.1556 & 1.0312 & 1.2950 \\
\hline dyslipidemia & 0.2913 & 0.0484 & 6.02 & $<.0001$ & 1.3382 & 1.2170 & 1.4712 \\
\hline hyperglycemia & -0.0503 & 0.0778 & -0.65 & 0.5176 & 0.9509 & 0.8164 & 1.1075 \\
\hline time & -0.0117 & 0.0191 & -0.61 & 0.5417 & 0.9884 & 0.9520 & 1.0261 \\
\hline baseage & 0.0055 & 0.0023 & 2.42 & 0.0155 & 1.0055 & 1.0010 & 1.0101 \\
\hline GGT & 0.0061 & 0.0013 & 4.86 & $<.0001$ & 1.0061 & 1.0036 & 1.0085 \\
\hline ALB & -0.044 & 0.0096 & -4.56 & $<.0001$ & 0.9570 & 0.9390 & 0.9752 \\
\hline GLO & -0.0075 & 0.0063 & -1.2 & 0.2319 & 0.9925 & 0.9805 & 1.0048 \\
\hline $\mathrm{CHOL}$ & 0.0115 & 0.0269 & 0.43 & 0.6702 & 1.0116 & 0.9595 & 1.0663 \\
\hline $\mathbf{H b}$ & 0.0188 & 0.0028 & 6.81 & $<.0001$ & 1.0190 & 1.0135 & 1.0245 \\
\hline MCV & -0.0147 & 0.0109 & -1.34 & 0.179 & 0.9854 & 0.9646 & 1.0067 \\
\hline $\mathrm{MCH}$ & -0.0155 & 0.0264 & -0.59 & 0.5574 & 0.9846 & 0.9349 & 1.0370 \\
\hline WBC & 0.0597 & 0.0162 & 3.68 & 0.0002 & 1.0615 & 1.0283 & 1.0959 \\
\hline
\end{tabular}

Table 5 The results of multiple GEE analysis for UA levels and hypertension after adjusting other potential confounding factors

\begin{tabular}{|c|c|c|c|c|c|c|c|}
\hline & Estimate & Error & $z$ & $\operatorname{Pr}>|Z|$ & RR & $\begin{array}{l}\text { lower } 95 \% \\
\text { Confidence } \\
\text { Limits }\end{array}$ & $\begin{array}{l}\text { upper } 95 \% \\
\text { Confidence } \\
\text { Limits }\end{array}$ \\
\hline Intercept & -5.9514 & 1.0022 & -5.94 & $<.0001$ & & & \\
\hline Q4 & 0.3953 & 0.0822 & 4.81 & $<.0001$ & 1.4848 & 1.2639 & 1.7444 \\
\hline Q3 & 0.1751 & 0.0804 & 2.18 & 0.0294 & 1.1914 & 1.0178 & 1.3947 \\
\hline Q2 & -0.0189 & 0.0808 & -0.23 & 0.8146 & 0.9813 & 0.8375 & 1.1496 \\
\hline Q1 & 0 & 0 & ref & ref & ref & ref & ref \\
\hline hyperglycemia & 0.345 & 0.0843 & 4.09 & $<.0001$ & 1.4120 & 1.1969 & 1.6658 \\
\hline dyslipidemia & -0.3991 & 0.0596 & -6.69 & $<.0001$ & 0.6709 & 0.5969 & 0.7541 \\
\hline obesity & 0.1882 & 0.0584 & 3.22 & 0.0013 & 1.2071 & 1.0766 & 1.3534 \\
\hline time & 0.101 & 0.0218 & 4.64 & $<.0001$ & 1.1063 & 1.0600 & 1.1544 \\
\hline baseage & 0.0686 & 0.0027 & 25.55 & $<.0001$ & 1.0710 & 1.0655 & 1.0767 \\
\hline GGT & 0.0077 & 0.001 & 7.62 & $<.0001$ & 1.0077 & 1.0057 & 1.0097 \\
\hline ALB & 0.0028 & 0.0119 & 0.23 & 0.8149 & 1.0028 & 0.9797 & 1.0263 \\
\hline GLO & 0.0155 & 0.0071 & 2.19 & 0.0285 & 1.0156 & 1.0016 & 1.0298 \\
\hline $\mathrm{CHOL}$ & 0.0216 & 0.0314 & 0.69 & 0.4923 & 1.0218 & 0.9608 & 1.0867 \\
\hline $\mathbf{H b}$ & 0.0135 & 0.0031 & 4.31 & $<.0001$ & 1.0136 & 1.0074 & 1.0198 \\
\hline MCV & -0.0276 & 0.0131 & -2.1 & 0.0357 & 0.9728 & 0.9481 & 0.9982 \\
\hline $\mathrm{MCH}$ & 0.0114 & 0.032 & 0.36 & 0.722 & 1.0115 & 0.9500 & 1.0768 \\
\hline WBC & 0.0538 & 0.0186 & 2.8900 & 0.0039 & 1.0553 & 1.0175 & 1.0945 \\
\hline
\end{tabular}


adolescents [14], in an urban population in Turkey [15], and in many other populations. Particularly, the debate on whether UA should be viewed even as an additional component of MetS received intensive discussions [16]; and the relationship between UA and each MetS component was also controversial. In this paper, based on a large longitudinal cohort study over 6 years, we confirmed that UA level is positively and significantly associated with risk of MetS among healthy male adults in Han Chinese of Shandong province, after adjusting other potential confounding factors using GEE regression analyses. This provides us a strong piece of evidence for developing risk assessment model for early screening of MetS.

As an essential component of MetS, obesity was regarded as a main contributor of the increasing prevalence of MetS, because obesity was proved as a risk factor of hypertension, dyslipidemia and hyperuricemia [17]. The positive gradient of obesity with increasing UA level has been found among several recent studies $[18,19]$. In this study, we found consistent results: in Chinese Han urban male population from middle to upper socioeconomic classes, UA levels of Q2, Q3, Q4 correspond to relative risks of 1.53, 2.04, and 2.51, respectively, in getting obesity (with Q1 as reference level; see Table 7. Additionally we found that the effect of UA on obesity become much larger, compared to MetS; see Table 3 . In pathophysiology, it was explained in three ways for the fact that obesity causes higher level of UA in blood: (1) obesity directly interferes with urate synthesis and excretion [20]; (2) obesity causes renal damage via glomerulus dysfunction [21]; (3) obesity leads to dysfunction of the reninangiotensin system, which would eventually results in fractional clearance of UA [22].

The association between hyperglycemia and UA, however, still remained controversy. Most researches suggested that hyperglycemia and UA should be the pathophysiological underpinning of MetS a long time ago [23], and a number of prospective studies have provided evidence that individuals with higher UA are at a higher risk of diabetes $[9,24,25]$. Nonetheless, some studies claimed that UA is negatively associated with diabetes in Asian men [7, 12], and an inverse association between UA and fasting plasma glucose was also observed in adult residents of Taiwan [6]. In our study, also in Asian male population, Q2-Q4 of UA was found to have lower risk of hyperglycemia with adjusted OR from 0.58-0.74; see Table 5 . This protective effect can be explained in two ways: (1) UA-hyperglycemia association might be gender-dependent among Asian populations (i.e., UA is a risk factor in females, but a protective factor in males); (2) the relationship might be nonlinear and hyperglycemia could impair tubular reabsorption of UA [6, 26].

UA was first demonstrated to be associated with hypertension by Mohamed in $1870 \mathrm{~s}$ [27], and in 2008, based on the large population in the Normative Aging Study. The

Table 6 The results of multiple GEE analysis for UA levels and dyslipidemia after adjusting other potential confounding factors

\begin{tabular}{|c|c|c|c|c|c|c|c|}
\hline & Estimate & Error & $Z$ & $\operatorname{Pr}>|Z|$ & RR & $\begin{array}{l}\text { lower } 95 \% \\
\text { Confidence } \\
\text { Limits }\end{array}$ & $\begin{array}{l}\text { upper } 95 \% \\
\text { Confidence } \\
\text { Limits }\end{array}$ \\
\hline Intercept & -0.4838 & 0.8527 & -0.57 & 0.5704 & & & \\
\hline Q4 & 0.4752 & 0.0713 & 6.66 & $<.0001$ & 1.6083 & 1.3985 & 1.8498 \\
\hline Q3 & 0.1815 & 0.067 & 2.71 & 0.0068 & 1.1990 & 1.0514 & 1.3672 \\
\hline Q2 & 0.0526 & 0.0659 & 0.8 & 0.4247 & 1.0540 & 0.9264 & 1.1993 \\
\hline Q1 & 0 & 0 & ref & ref & ref & ref & ref \\
\hline hyperglycemia & 0.1725 & 0.0795 & 2.17 & 0.03 & 1.1883 & 1.0168 & 1.3886 \\
\hline hypertension & -0.4309 & 0.0603 & -7.15 & $<.0001$ & 0.6499 & 0.5775 & 0.7315 \\
\hline obesity & 0.2916 & 0.0485 & 6.02 & $<.0001$ & 1.3386 & 1.2173 & 1.4720 \\
\hline time & -0.23 & 0.0196 & -11.75 & $<.0001$ & 0.7945 & 0.7646 & 0.8256 \\
\hline baseage & -0.009 & 0.0023 & -3.86 & 0.0001 & 0.9910 & 0.9865 & 0.9956 \\
\hline GGT & 0.0106 & 0.0013 & 8.39 & $<.0001$ & 1.0107 & 1.0081 & 1.0131 \\
\hline ALB & 0.0005 & 0.01 & 0.05 & 0.9605 & 1.0005 & 0.9810 & 1.0204 \\
\hline GLO & 0.0101 & 0.0063 & 1.59 & 0.1109 & 1.0102 & 0.9977 & 1.0229 \\
\hline CHOL & -0.0465 & 0.027 & -1.72 & 0.085 & 0.9546 & 0.9054 & 1.0064 \\
\hline $\mathbf{H b}$ & 0.0108 & 0.0027 & 3.96 & $<.0001$ & 1.0109 & 1.0055 & 1.0163 \\
\hline MCV & -0.097 & 0.0114 & -8.51 & $<.0001$ & 0.9076 & 0.8875 & 0.9281 \\
\hline MCH & 0.2093 & 0.0278 & 7.53 & $<.0001$ & 1.2328 & 1.1674 & 1.3017 \\
\hline WBC & 0.163 & 0.0166 & 9.8 & $<.0001$ & 1.1770 & 1.1393 & 1.2160 \\
\hline
\end{tabular}


Table 7 The results of multiple GEE analysis for UA levels and hyperglycemia after adjusting other potential confounding factors

\begin{tabular}{llllllll}
\hline Quartiles & Estimate & Error & $\mathbf{Z}$ & $\operatorname{Pr}>|Z|$ & RR & $\begin{array}{l}\text { lower } 95 \% \\
\text { Confidence Limits }\end{array}$ & $\begin{array}{l}\text { upper } \mathbf{9 5} \% \\
\text { Confidence Limits }\end{array}$ \\
\hline Intercept & -3.9156 & 1.2888 & -3.04 & 0.0024 & & & \\
Q4 & $-\mathbf{0 . 3 7 5 2}$ & $\mathbf{0 . 1 0 6 9}$ & $\mathbf{- 3 . 5 1}$ & $\mathbf{0 . 0 0 0 4}$ & $\mathbf{0 . 6 8 7 2}$ & $\mathbf{0 . 5 5 7 3}$ & $\mathbf{0 . 8 4 7 3}$ \\
Q3 & $-\mathbf{0 . 5 4 2 7}$ & $\mathbf{0 . 1 0 8 4}$ & $-\mathbf{5 . 0 1}$ & $<.0001$ & $\mathbf{0 . 5 8 1 2}$ & $\mathbf{0 . 4 7 0 0}$ & $\mathbf{0 . 7 1 8 8}$ \\
Q2 & $-\mathbf{0 . 3 0 6 3}$ & $\mathbf{0 . 1 0 2 6}$ & $\mathbf{- 2 . 9 8}$ & $\mathbf{0 . 0 0 2 8}$ & $\mathbf{0 . 7 3 6 2}$ & $\mathbf{0 . 6 0 2 1}$ & $\mathbf{0 . 9 0 0 2}$ \\
Q1 & 0 & 0 & ref & ref & ref & ref & ref \\
hypertension & $\mathbf{0 . 3 2 6 9}$ & $\mathbf{0 . 0 8 7 5}$ & $\mathbf{3 . 7 4}$ & $\mathbf{0 . 0 0 0 2}$ & $\mathbf{1 . 3 8 6 7}$ & $\mathbf{1 . 1 6 8 1}$ & $\mathbf{1 . 6 4 6 1}$ \\
dyslipidemia & $\mathbf{0 . 1 6 7 2}$ & $\mathbf{0 . 0 7 9 9}$ & $\mathbf{2 . 0 9}$ & $\mathbf{0 . 0 3 6 3}$ & $\mathbf{1 . 1 8 2 0}$ & $\mathbf{1 . 0 1 0 8}$ & $\mathbf{1 . 3 8 2 2}$ \\
obesity & 0.007 & 0.0774 & 0.09 & 0.9277 & 1.0070 & 0.8652 & 1.1721 \\
time & $\mathbf{0 . 2 3 5 2}$ & $\mathbf{0 . 0 2 7 9}$ & $\mathbf{8 . 4 3}$ & $<.0001$ & $\mathbf{1 . 2 6 5 2}$ & $\mathbf{1 . 1 9 7 8}$ & $\mathbf{1 . 3 3 6 3}$ \\
baseage & $\mathbf{0 . 0 3 2 7}$ & $\mathbf{0 . 0 0 3 3}$ & $\mathbf{1 0 . 0 3}$ & $<.0001$ & $\mathbf{1 . 0 3 3 2}$ & $\mathbf{1 . 0 2 6 6}$ & $\mathbf{1 . 0 3 9 9}$ \\
GGT & $\mathbf{0 . 0 0 5 6}$ & $\mathbf{0 . 0 0 1}$ & $\mathbf{5 . 5 9}$ & $<.0001$ & $\mathbf{1 . 0 0 5 6}$ & $\mathbf{1 . 0 0 3 6}$ & $\mathbf{1 . 0 0 7 6}$ \\
ALB & 0.0218 & 0.016 & 1.36 & 0.1735 & 1.0220 & 0.9904 & 1.0545 \\
GLO & -0.0014 & 0.0093 & -0.15 & 0.8834 & 0.9986 & 0.9805 & 1.0170 \\
CHOL & $\mathbf{0 . 2 5 0 7}$ & $\mathbf{0 . 0 4 0 5}$ & $\mathbf{6 . 1 9}$ & $<.0001$ & $\mathbf{1 . 2 8 4 9}$ & $\mathbf{1 . 1 8 6 8}$ & $\mathbf{1 . 3 9 1 1}$ \\
Hb & $-\mathbf{0 . 0 1 0 1}$ & $\mathbf{0 . 0 0 4 1}$ & $\mathbf{- 2 . 4 7}$ & $\mathbf{0 . 0 1 3 6}$ & $\mathbf{0 . 9 9 0 0}$ & $\mathbf{0 . 9 8 2 0}$ & $\mathbf{0 . 9 9 7 9}$ \\
MCV & $\mathbf{0 . 0 9 3 5}$ & $\mathbf{0 . 0 1 8 6}$ & $\mathbf{- 5 . 0 4}$ & $<.0001$ & $\mathbf{0 . 9 1 0 7}$ & $\mathbf{0 . 8 7 8 2}$ & $\mathbf{0 . 9 4 4 5}$ \\
MCH & $\mathbf{0 . 2 2 3 3}$ & $\mathbf{0 . 0 4 3 6}$ & $\mathbf{5 . 1 2}$ & $<.0001$ & $\mathbf{1 . 2 5 0 2}$ & $\mathbf{1 . 1 4 7 6}$ & $\mathbf{1 . 3 6 1 8}$ \\
WBC & $\mathbf{0 . 0 7 5 3}$ & $\mathbf{0 . 0 2 3 6}$ & $\mathbf{3 . 1 9 0 0}$ & $\mathbf{0 . 0 0 1 4}$ & $\mathbf{1 . 0 7 8 2}$ & $\mathbf{1 . 0 2 9 4}$ & $\mathbf{1 . 1 2 9 3}$ \\
\hline & & & & & &
\end{tabular}

baseline UA was reported as a durable marker of risk of hypertension [28]. In our longitudinal cohort study with 6 years of follow up, the GEE analyses confirmed that UA is an independent risk factor of hypertension in Chinese Han urban males; see Table 6. As found by Watanabe et al. [29], once elevated UA level causes sufficient renal injury, human body would develop salt-sensitive hypertension. Elevated UA also associates with increased amount of free radicals [30] and an oxidative stress, which may further abolish endothelium-dependent vasodilatation [31].

Finally, it has been well received that elevated UA level would increase the risk of dyslipidemia [12, 30]. Our longitudinal study also suggests a positive association between UA dyslipidemia; see Table 4. Although the role of UA in the metabolism of triglycerides and other lipids still remains unclear, it was believed that UA might be involved in either the overproduction or the reduction of clearance of lipids [32].

\section{Conclusions}

Association between UA and cardiovascular diseases has been reported frequently recent years. Many research confirmed that UA was a strong risk factor for cardiovascular diseases, especially MetS [33-35]. Our longitudinal study further verified that UA is an independent risk factor of MetS, higher level of UA leading to higher risk of MetS. All the analyses were based on GEE regression models with data from the longitudinal cohort study with Han
Chinese urban male participants who were from middle to upper socioeconomic classes. Additionally, our study suggested that UA level be an independent risk factor to obesity, hypertension and dyslipidemia, but a protective factor to hyperglycemia. These findings are concordant with results from other studies on Asian populations, and jointly provide a basis to further develop a risk assessment model for predicting MetS using UA levels and other factors in China.

There were several limitations in the present study. Due to the fact that participants of the study who came to the hospitals mainly for physical examinations, they were mandatorily asked to report histories on medication for treating MetS or related medical disorders (e.g., hypertension). Thus we did not have data on medication in our GEE regression analyses in studying the UA-MetS association. Also considering that our study contains only local males from relatively rich and educated families in Shandong province, and the fact that our analyses only covered a follow-up period of 6 years and no genetic information was included, it is expected a large scale longitudinal study is expected for a better and fully understanding of the target relationships.

\section{Additional file}

Additional file 1: Table S1. The distribution of UA levels and other potential confounding factors. Table S2 Numbers Participants at Each Year of the Study 


\section{Competing interests}

There is no conflict of interest for any of the authors. All authors had access to the data and were involved in drafting the article and revising it critically for important intellectual content.

\section{Author details}

${ }^{1}$ Department of Epidemiology and Health Statistics, School of Public Health, Shandong University, PO Box 100, Jinan 250012, China. ${ }^{2}$ Health Management Center, Shandong Provincial QianFoShan Hospital, Jinan 250014, China. ${ }^{3}$ Center for Health Management, Provincial Hospital affiliated to Shandong University, Jinan, China. ${ }^{4}$ Department of Epidemiology and Biostatistics, Drexel University School of Public Health, Philadelphia, PA, USA. ${ }^{5}$ Department of Public Health Sciences, School of Medicine, University of California, Davis, Davis, CA 95616, USA.

\section{Authors' contributions}

In our work, FX and CZ designed the study and directed its implementation, including quality assurance and control. XS, FT, HL and DZ did the clinical exam and collected the data. WM, YZ and ZZ helped analyzing the data. LL provided scientific comments and advice. XY worked on writing of the paper. QZ participated much of the above work and led the writing of the paper. All authors read and approved the final manuscript

Received: 28 October 2011 Accepted: 8 June 2012

Published: 8 June 2012

\section{References}

1. Grundy SM, Brewer HB Jr, Cleeman Jl, et al: Definition of metabolic syndrome: Report of the National Heart, Lung, and Blood Institute/ American Heart Association conference on scientific issues related to definition. Circulation 2004, 109(3):433-438. Jan 27.

2. Wilson PW, D'Agostino RB, Parise $H$, et al: Metabolic syndrome as a precursor of cardiovascular disease and type 2 diabetes mellitus. Circulation 2005, 112(20):3066-3072. Nov 15.

3. Kang YH, Min HG, Kim IJ, Kim YK, Son SM: Comparison of alanine aminotransferase, white blood cell count, and uric acid in their association with metabolic syndrome: a study of Korean adults. Endocr J. 2008, 55(6):1093-1102. Dec.

4. Koulouridis E, Georgalidis K, Kostimpa I, Koulouridis I, Krokida A, Houliara D: Metabolic syndrome risk factors and estimated glomerular filtration rate among children and adolescents. Pediatr Nephrol 2010, 25(3):491-498.

5. Chang CH, Chen YM, Chuang YW, Liao SC, Lin CS, Tang YJ, Sheu WH, Chen DY: Relationship between hyperuricemia (HUC) and metabolic syndrome (MS) in institutionalized elderly men. Arch Gerontol Geriatr 2009, 49(Suppl 2):S46-S49. Dec.

6. Liu PW, Chang TY, Chen JD: Serum uric acid and metabolic syndrome in Taiwanese adults. Metabolism 2010, 59(6):802-807.

7. Lin YC, Chen JD, Lo SH, Chen PC: Worksite health screening programs for predicting the development of Metabolic Syndrome in middle-aged employees: a five-year follow-up study. BMC Public Health 2010, 10:747.

8. Sui X, Church TS, Meriwether RA, Lobelo F, Blair SN: Uric acid and the development of metabolic syndrome in women and men. Metabolism 2008, 57(6):845-852.

9. Lu YH, Lu JM, Wang SY, Li CL, Liu LS, Zheng RP, Tian H, Wang XL, Yang $\sqcup$, Zhang YQ, Pan CY: Comparison of the diagnostic criteria of metabolic syndrome by International Diabetes Federation and that by Chinese Medical Association Diabetes Branch. Zhonghua Yi Xue Za Zhi 2006, 86(6):386-389.

10. Schafer JL: Analysis of incomplete multivariate data. London: Chapman and Hall; 1997.

11. Choi HK, Ford ES: Prevalence of the metabolic syndrome in individuals with hyperuricemia. Am J Med 2007, 120(5):442-447.

12. Ryu S, Song J, Choi BY, Lee SJ, Kim WS, Chang Y, Kim DI, Suh BS, Sung KC: Incidence and risk factors for metabolic syndrome in Korean male workers, ages 30 to 39. Ann Epidemiol 2007, 17(4):245-252.

13. Lohsoonthorn V, Dhanamun B, Williams MA: Prevalence of hyperuricemia and its relationship with metabolic syndrome in Thai adults receiving annual health exams. Arch Med Res 2006, 37(7):883-889.

14. Huang RC, Mori TA, Burke V, Newnham J, Stanley FJ, Landau LI, Kendall GE Oddy WH, Beilin LJ: Synergy between adiposity, insulin resistance, metabolic risk factors, and inflammation in adolescents. Diabetes Care 2009, 32(4):695-701.
15. Sari I, Akar S, Pakoz B, Sisman AR, Gurler O, Birlik M, Onen F, Akkoc N: Hyperuricemia and its related factors in an urban population, Izmir, Turkey. Rheumatol Int 2009, 29(8):869-874.

16. Sheu $\mathrm{WH}$, Tseng $\mathrm{YH}$ : Uric acid: an additional component of metabolic syndrome? J Chin Med Assoc 2006, 69(3):99-100.

17. Expert Panel on Detection, Evaluation and treatment of high blood cholesterol in adults. JAMA 2001, 285:2486-2497. Executive summary of the Third Report of the National Cholesterol Education Program (NCEP) Expert Panel on Detection, Evaluation, and Treatment of High Blood Cholesterol in Adults (Adult Treatment Panel III).

18. Lin JD, Chiou WK, Chang HY, Liu FH, Weng HF: Serum uric acid and leptin levels in metabolic syndrome: a quandary over the role of uric acid. Metabolism 2007, 56:751-756.

19. Schmidt MI, Watson RL, Duncan BB, et al: Clustering of dyslipidemia, hyperuricemia, diabetes, and hypertension and its association with fasting insulin and central and overall obesity in a general population. Atherosclerosis Risk in Communities Study Investigators. Metabolism 1996, 45:699-706.

20. Marasini B, Massarotti M: What rheumatologists should know about gout and cardiovascular disease. J Rheumatol 2009, 36:854-855.

21. Tuttle KR: Renal manifestations of the metabolic syndrome. Nephrol Dial Transplant 2005, 20:861-864.

22. Indraratna PL, Williams KM, Graham GG, Day RO: Hyperuricemia, cardiovascular disease, and the metabolic syndrome. J Rheumatol 2009, 36(12):2842-2843. author reply 2844.

23. Facchini F, Chen YD, Hollenbeck CB, Reaven GM: Relationship between resistance to insulin-mediated glucose uptake, urinary uric acid clearance, and plasma uric acid concentration. JAMA 1991, 266:3008-3011.

24. Bhole V, Choi JW, Kim SW, de Vera M, Choi H: Serum uric acid levels and the risk of type 2 diabetes: a prospective study. Am J Med 2010, 123 (10):957-961.

25. Niskanen L, Laaksonen DE, Lindstrom J, et al: Serum uric acid as a harbinger of metabolic outcome in subjects with impaired glucose tolerance: the Finnish Diabetes Prevention Study. Diabetes Care 2006, 29:709-711.

26. Lin KC, Tsai ST, Lin HY, et al: Different progressions of hyperglycemia and diabetes among hyperuricemic men and women in the Kinmen study. $J$ Rheumatol 2004, 31:1159-1165.

27. Mohamed FA: On chronic Bright's disease, and its essential symptoms. Lancet 1879, 1:399-401.

28. Perlstein TS, Gumieniak O, Williams GH, Sparrow D, Vokonas PS, Gaziano M, Weiss ST, Litonjua AA: Uric acid and the development of hypertension: the normative aging study. Hypertension 2006, 48(6):1031-1036. Epub 2006 Oct 23.

29. Watanabe S, Kang DH, Feng L, Nakagawa T, Kanellis J, Lan H, Mazzali M, Johnson RJ: Uric acid, hominoid evolution, and the pathogenesis saltsensitivity. Hypertension 2002, 40:355-360.

30. Vasquez-Vivar J, Santos AM, Junqueira VB, et al: Peroxynitritemediated formation of free radicals in human plasma: EPR detection of ascorbyl, albuminthiyl and uric acid-derived free radicals. Biochem J 1996, 314:869-876.

31. Tsouli SG, Liberopoulos EN, Mikhailidis DP, Athyros VG, Elisaf MS: Elevated serum uric acid levels in metabolic syndrome: an active component or an innocent bystander? Metabolism 2006, 55(10):1293-1301.

32. Nakagawa T, Hu H, Zharikov S, Tuttle KR, Short RA, Glushakova O, Ouyang X, Feig DI, Block ER, Herrera-Acosta J, Patel JM, Johnson RJ: A causal role for uric acid in fructose-induced metabolic syndrome. Am J Physiol Renal Physiol 2006, 290(3):F625-F631.

33. de Oliveira EP, Burini RC: High plasma uric acid concentration: causes and consequences. Diabetol Metab Syndr 2012, 4(1):12.

34. Santos RD: Elevated uric acid, the metabolic syndrome and cardiovascular disease: cause, consequence, or just a not so innocent bystander?. Endocrine 2012, 41(3):350-352.

35. Kackov S, Simundić AM, Nikolac N, Bilusić M: The association of uric acid with glucose and lipids in general population: Croatian cross-sectional study. Coll Antropol 2011, 35(4):1055-1059.

doi:10.1186/1471-2458-12-419

Cite this article as: Zhang et al:: A longitudinal cohort based association study between uric acid level and metabolic syndrome in Chinese Han urban male population. BMC Public Health 2012 12:419. 